\title{
Herztod-Alarm bei Androgenentzug: Entwarnung
}

\section{Hoch kontrovers diskutiert: Treibt die Androgendeprivation bei Patienten mit Prostatakarzinom die kardiovaskuläre Mortalität in die Höhe? Nach Alarmmeldungen der letzten Jahre sorgt eine neue Metaanalyse für Beruhigung.}

$\mathrm{D}$ ie Androgendeprivationstherapie (ADT) mit Agonisten des Gonadotropin-Releasing-Hormons $(\mathrm{GnRH})$ ist eine wichtige Säule in der Behandlung des fortgeschrittenen Prostatakarzinoms. Weil vor allem in retrospektiven Studien eine Zunahme der kardiovaskulären Mortalität auftrat, mahnen einige US-Fachgesellschaften zur Vorsicht, und die Food and Drug Administration (FDA) hat einen entsprechenden Warnhinweis für GnRHAgonisten veranlasst.

Eine Metaanalyse von acht prospektiv randomisierten Studien mit 4.141 Patienten bringt jetzt mehr Klarheit. Berücksichtigt wurden nur Studien, die sofortige GnRH-Agonisten-basierte ADT gegen keine oder verzögerte ADT bei Patienten mit nicht metastasiertem, aber prognostisch ungünstigem Prostatakarzinom unter die Lupe nahmen, vollständige Informationen über kardiovaskuläre Todesfälle und eine mediane Beobachtungszeit von mehr als einem Jahr aufwiesen.

Die Zahl kardiovaskulärer Todesfälle zwischen ADT-und Kontroll-Patienten unterschied sich mit einer Inzidenz von $11,0 \%$ vs. $11,2 \%$ nicht signifikant. Ähnliches galt für die Studien mit einer über mindestens drei Jahre (11,5\% vs. $11,5 \%)$ beziehungsweise nur maximal sechs Monate laufenden ADT (10,5\% vs. 10,3\%). Die Analyse von 4.805 Patienten aus elf
Studien mit Daten zur Gesamtmortalität ergab, dass sofortiger Androgenentzug mit signifikant geringerer Prostatakarzinom-spezifischer (13,5\% vs. $22,1 \%)$ und Gesamtmortalität (37,7\% vs. $44,4 \%)$ einherging.

Fazit: Diese Metaanalyse fand keine Evidenz, dass Androgenentzug das Risiko für kardiovaskuläre Todesfälle bei Prostatakarzinom-Patienten steigert. Die Patienten profitierten jedoch beim Prostatakarzinom-spezifischen und Gesamtüberleben. Unklar bleibt, ob man auch für Patienten mit chronischer Herzinsuffizienz oder Zustand nach Myokardinfarkt Entwarnung geben kann.

Ulrike Wepner

Nguyen PL et al. Association of androgen deprivation therapy with cardiovascular death in patients with prostate cancer. JAMA 2011; 306: 2359-66

\section{Androgendeprivation mit Histrelin: Langzeitdaten veröffentlicht}

In der Histrelin-Zulassungsstudie konnte belegt werden, dass sich mit dem GnRH-Agonist die Testosteronspiegel dauerhaft auf Kastrationsniveau halten lassen. Die Erweiterungsstudie sollte zeigen, ob das auch über einen Zeitraum von mehreren Jahren gelingt.

Z el der Androgendeprivation ist, den Testosteronspiegel dauerhaft auf Kastrationsniveau (mindestens unter $50 \mathrm{ng} /$ dl) zu senken, um das Wachstum hormonabhängiger Prostatatumoren zu verlangsamen. Histrelin ist ein synthetisches Analogon des GnRH („gonadotropinreleasing hormone"), das subkutan in Form eines Hydrogel-Depots implantiert wird und für einen Zeitraum von zwölf Monaten kontinuierlich seinen Wirkstoff ins Gewebe freigibt. Danach muss es ausgetauscht werden. In der Zulassungsstudie über 60 Wochen wurden Wirkung und Verträglichkeit von Histrelin belegt. In einer Extensionsstudie wurde nun untersucht, ob sich die Testosteronspiegel auch über einen Zeitraum von mehreren Jahren unter Kastrationsniveau halten lassen.
104 Patienten erhielten ab Woche 104 ein neues Histrelin-Implantat und in der Folge alle 52 Wochen. Alle drei Monate wurden Testosteron- und PSA-Werte sowie Nebenwirkungen und Analgetikaverbrauch registriert. Die Studie wurde nach 51 Monaten, zum Zeitpunkt der Zulassung in den USA, beendet.

Die durchschnittlichen Testosteronwerte blieben über den kompletten Studienzeitraum im Bereich zwischen 10,8 und $20,8 \mathrm{ng} / \mathrm{dl}$. Nach einem Implantatwechsel traten keine Testosteron-Surges auf. Nur bei einem Patienten wurde ein Wert über $50 \mathrm{ng} / \mathrm{dl}$ gemessen - nachdem das Implantat ausgetauscht wurde, sank der Wert wieder unter $20 \mathrm{ng} / \mathrm{dl}$.

Die häufigsten therapiebedingten $\mathrm{Ne}$ benwirkungen waren Hitzewallungen
(64,4\% der Patienten), Fatigue (7,7\%), Hodenatrophie $(7,7 \%)$ und Gynäkomastie $(4,8 \%)$. Zwei Patienten verlangten die Herausnahme des Histrelin-Implantats - einer aufgrund von Hitzewallungen, der andere aufgrund von Schmerzen an der Implantatstelle. Nach der Entfernung war die Nebenwirkung aufgehoben. Die Autoren sehen darin einen klinischen Vorteil der Galenik. Im Gegensatz zu Injektionen könne das Hydrogel-Depot bei Nebenwirkungen einfach wieder entfernt werden.

Fazit: In der Verlängerungsstudie wurden die Ergebnisse der Zulassungsstudie bestätigt. Mit dem GnRH-Agonist Histrelin konnten die Serumtestosteronwerte dauerhaft unter $20 \mathrm{ng} / \mathrm{dl}$ gehalten werden.

Sebastian Lux

Shore $\mathrm{N}$ et al. Long-term efficacy and tolerability of once-yearly histrelin acetate subcutaneous implant in patients with advanced prostate cancer. BJU Int 2012; 109, 226-32 